\title{
Accuracy of CBCT based computer assisted surgical guide for implant placement with tilted position in posterior atrophied mandible \\ Original
} Article
Enas Anter Abdel Ghafar ${ }^{a}$ and Ghada Abdel Monim ${ }^{b}$

${ }^{a}$ Department of Oral and Maxillofacial Radiology, ${ }^{b}$ Department of Oral and Maxillofacial, Faculty of Dentistry, Cairo University, Cairo, Egypt

\begin{abstract}
Purpose: Investigate the accuracy of CBCT based computer aided surgical guide CASG for implant placement in posterior atrophied mandible with tilted position (buccal or lingual) to inferior alveolar nerve IAN avoiding its injury.

Materials and Methods: 15 partially edentulous patients with atrophied mandible were included based on certain eligibility criteria, the work flow in this study run as following, CBCT imaging, virtual implant planning and CASG designing, 3D stereolithographic printing of surgical guide, surgical procedures of implant placement using the CASG, post-operative CBCT imaging, registration of pre- and post-operative CBCT images, assessment of angular deviation between actual and planned implants and linear deviation between them cervically and apically, comparison between virtual and actual implants distance to IAN.

Results: Wilcoxon signed-rank test Comparing between virtual and actual implants' distance to IAN showed a statistically significant difference with a mean distance of $0.72 \pm 0.25 \mathrm{~mm}$ for actual implants and $1.7 \pm 0.56 \mathrm{~mm}$ for virtual implants. $\left(4.13 \pm 1.86^{\circ}\right)$ was the mean angular deviation, while $1.15 \pm 0.49 \mathrm{~mm}$ and $1.32 \pm 0.63$ were the mean cervical and apical linear deviation respectively.

Conclusion: although a significant difference was found between virtual and actual implants distance to IAN, the values of linear and angular deviation between them were well accepted and within the range reported by most of similar clinical studies with less crucial situations, which enhance using fully guided stereolithographic CASG for implant placement with tilted position in posterior atrophied mandible but with considering at least $2 \mathrm{~mm}$ distance between virtual implant and IAN in planning procedures.
\end{abstract}

Key Words: Atrophied Mandible, By-pass- Computer aided surgical guide, CBCT, Dental Implants, Inferior Alveolar Nerve.

Received: 06 February 2021, Accepted: 01 March 2021.

Corresponding Author: Enas Anter Abdel Ghafar, Department of Oral and Maxillofacial Radiology, Faculty of Dentistry, Cairo University, Cairo, Egypt, Tel.: +20227120175, Mobile: +201006276186, E-mail: enas.anter@dentistry.cu.edu.eg.

ISSN: 2090-097X, July 2020, Vol. 11, No. 3

\section{INTRODUCTION}

Rehabilitation of posterior edentulous severely atrophied mandible is considered a real challenge, as minimal bone superior to inferior alveolar nerve 'IAN' is left reducing the volume of implantable bone in this site ${ }^{[1]}$. In case when inadequate bone exists, restorative options might include the use of short implant with its questionable outcome in this site or shifting to surgical techniques to reconstruct the deficient ridge for implant placement, as guided bone regeneration with barrier membranes, bone grafts, ridge splitting and distraction osteogenesis ${ }^{[2-4]}$.

A different surgical solution for such a restorative dilemma has been tried by moving the IAN literally from its position through removing the buccal cortex surrounding the mandibular canal to allow for IAN repositioning either by lateral reflection posterior to the mental foramen without incisive nerve traction 'IAN Lateralization' or by trans-positioning both the mental foramen and the IAN in a way that the mental foramen is repositioned more posteriorly sacrificing the incisive neurovascular bundle 'IAN Transposition' ${ }^{[5,6]}$. These types of surgeries are made to allow for using implants with reasonable length without going for bone reconstructive surgeries that might not be possible, but unfortunately it was found be associated with high risk of neuropathies; as paresthesia, hypoesthesia or anesthesia of the IAN ${ }^{[7]}$.

The evolution of digital implant dentistry has started by introduction of CBCT imaging in dental field, allowing for $3 \mathrm{D}$ detailed preoperative evaluation of alveolar bone quantity and quality at the proposed implant site with accurate tracing of adjacent vital anatomical structures like $\mathrm{IAN}^{[8]}$. In sync with that, the massive development in computer technology both hardware and software has enabled the emersion of the recent digital systems that offered a great help in virtual planning for implant placement with prediction of the final surgical and prosthetic outcomes and moreover, it allows the accurate translation of the desired virtual treatment plane to the 
surgical theater via either direct navigation or computer assisted surgical guide (CASG) $)^{[8,9]}$.

CBCT and CASG allowed for what could be considered a safer option for IAN bypass in patient with atrophied mandible rather than going for IAN transposition or lateralization, as CBCT detailed evaluation of the residual alveolar ridge and accurate localization of IAN position permit planning implant placement in a tilted position either buccal or lingual to the IAN provided that a sufficient alveolar bone width is available $(5-6 \mathrm{~mm})$ not to perforate the lingual or buccal cortical plates, then using the CASG based on this virtual plan helps executing this trajectory ${ }^{[10-12]}$.

Does CASG provides an accurate transfer of the virtual plane to the operation theater or not? It is a question that has been dealt with in a number of previous studies, some of them found that the region where the implant is going to be placed has an effect on this accuracy ${ }^{[10,13,14]}$. For that this study was made to investigate the accuracy of CASG for implant placement in posterior atrophied mandible in a tilted position (either buccal or lingual) to IAN, where a high precision is badly needed to avoid the risk of IAN injury as a result of even minor inaccuracy in virtual plane transfer.

\section{MATERIALS AND METHODS}

This prospective observational clinical study was carried out after the approval of the Regional Research Ethics Committee on 15 partially edentulous patients who came to the outpatient clinic of Oral and Maxillofacial Surgery, Faculty of Dentistry, Cairo University seeking implant placement. Patients enrollment in this study was based on the following eligibility criteria:

- All the patients had an edentulous mandibular span posterior to the mental foramen.

- All the patients had a minimum of $8 \mathrm{~mm}$ of bone height above the IAN and alveolar bone width is not less than $6 \mathrm{~mm}$ (determined by the preoperative CBCT ${ }^{[11,12]}$.

- Both males and female patients were included.

- The IAN and its dominant region were free from any pathosis.

- No previous history of trauma or surgical operation at the intended site.

- No or minimal metallic restoration in the oral cavity (to avoid metallic artifacts in CBCT).

- Patients with inadequate inter-arch space, para-functional habits, uncontrolled diabetes, immunosuppressive diseases were all excluded.
This study was conducted between Jan 2019 and January 2020, the workflow in the study was done as following:

\section{I-Pre-operative procedures:}

\section{a-Clinical intra-oral and Extra-oral examination:}

After proper medical and dental history taking; clinical examination was carried out to reveal patients' hygiene, inter-arch distance, and presence of metallic dental restorations inside patient mouth.

\section{b- Radiographic Examination:}

- Firstly, panoramic radiograph was made as screening technique to preclude presence of pathosis at the proposed implant site and for primary assessment of the distance between the IAN and the implant site.

- Based on the panoramic radiographs' interpretation, the indicated cases underwent CBCT examination after fabrication of radiographic stents made of acrylic resin with Radio Opaque 'RO' markers -gutta percha pieces- attached at the proposed implant site. CBCT scanning was done using (Scanora 3DC, Soredex, Finland) machine, the exposure parameters used were, $12.5 \mathrm{~mA}, 90 \mathrm{Kv}$ with FOV $7 \mathrm{~cm} \times 14.5 \mathrm{~cm}$ and voxel size $0.2 \mathrm{~mm}$, then CBCT scanning of the patients' plaster casts with the radiographic stent was done with the same machine at the same exposure parameters (Dual scan technique), the CBCT images of the plaster dental casts were transferred in to STL file format.

\section{c- Virtual implant Planning:}

DICOM (Digital Imaging and Communications in Medicine) files of the CBCT and STL (Standard Tessellation Language) files of cast scans were imported in a treatment plan software "DDS-Pro, Czestochowa, Poland" where they were merged by point based registration depending on the anatomical landmarks of the teeth together with the RO markers on the radiographic stent creating a virtual patient model that was used for virtual implant simulation. (Figure 1 A).

Implant simulation was done with suitable sized implants such that the implant is at least $1 \mathrm{~mm}$ away from the inferior alveolar canal surrounded by at least $1 \mathrm{~mm}$ of bone bucco-lingually and at least $1.5 \mathrm{~mm}$ away from the inferior border of the mandible, the chosen virtual implants were placed with appropriate position in all 3 dimensions (Figures1 B and C). 


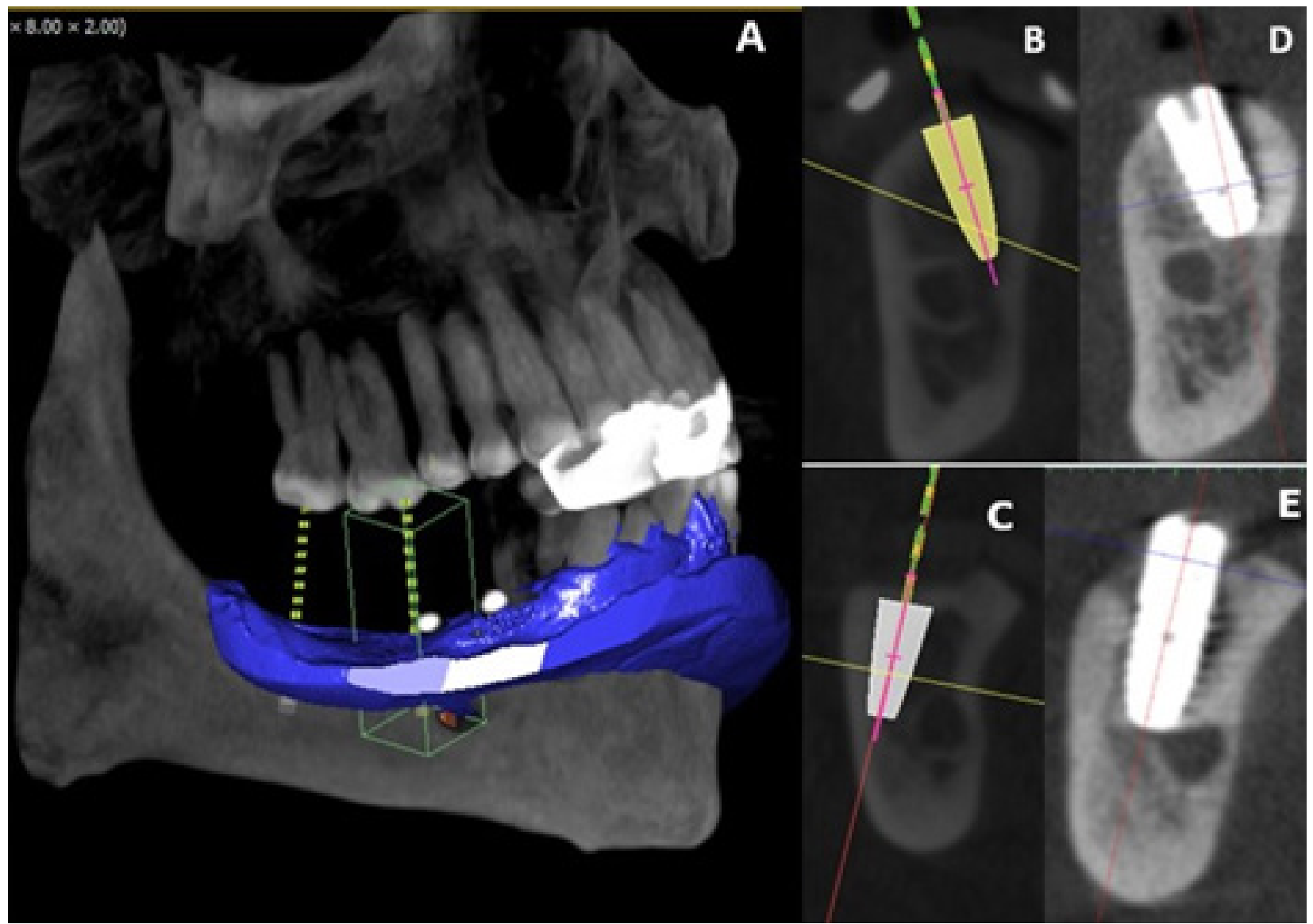

Figure 1: A. CBCT cast scan superimposed over CBCT patient's scan creating virtual patient model used for virtual implant planning. B and C. cross sectional CBCT images with virtual implants placed tilted in atrophied mandible for IAN bypass. D and E. post-operative CBCT images with the actual implants placed by CASG.

\section{d-Surgical guide designing and fabrication:}

After finalization of the virtual plane the same software 'DDS-Pro' was used for surgical guide designing, where teeth and mucosa supported surgical guide was planned in all the cases, the diameter of the guide tubes at the site of the implants was $2-3 \mathrm{~mm}$, the position for fixation pins was also incorporated (Figure $2 \mathrm{~A}$ ).

The virtual projects were then transferred to $1: 1$ scale model using rapid prototyping with stereolithography technology provided by "Formlabs Form 2 SLA 3D printer" using 'Clear Resin 1L' material, the generated surgical guides were checked on the stone cast to assess their fitness and stability, afterwards titanium sleeves 'Spectra, CA, USA.' were inserted through the drill guide holes in the 3D printed model (Figure $2 \mathrm{~B}$ ). Finally, was the stage of checking the surgical guide in the patient's mouth (Figure $2 \mathrm{C}$ ).

\section{e- Laboratory investigations:}

Complete blood count, renal function tests, liver function tests, International normalized ratio (INR) and blood sugar level were done for the selected patients prior to the surgical procedures.

\section{f-Informed consent:}

Prior to surgery, all patients were informed about the procedures they will go through and a written consent of each subject was obtained.

\section{II-Surgical procedures:}

After anesthetizing the patient with IAN block, lingual nerve block together with long buccal infiltration, the surgical guide was fixed inside the patient mouth by the fixative pins, flapless approach was adopted with a fully guided site preparation and implant insertion done using surgical guide kit: "Dentium, Hybrid design, 501 Gyeangyi, Korea.". after implant placement the surgical guide was removed then the covering screws were placed.

\section{III- Postoperative procedures:}

Following the surgical procedures, the patients underwent $\mathrm{CBCT}$ examination with the same exposure parameters used in pre-operative scanning for confirmation 
of the position of the actual implants in the mandible and their relation to the IAN (Figures 1 D, E and 3). firstly, the closest distance of the implant to the IAN were assessed both in the pre-operative $\mathrm{CBCT}$ with virtual implants and post-operative CBCT with actual implants (Figures $3 \mathrm{~A}$ and $\mathrm{D}$ ), then for each case the preoperative CBCT was registered to the postoperative CBCT after manual segmentation of the virtual and actual implants creating 3D images for both using 'DDS-Pro' software, images registration was based on the anatomical landmarks of the adjacent teeth.

Deviation between planned and actual implants was measured according to the method commonly used by previous researchers in this aera through assessment of the following measurements ${ }^{[10]}$ (Figure $3 \mathrm{E}$ ).
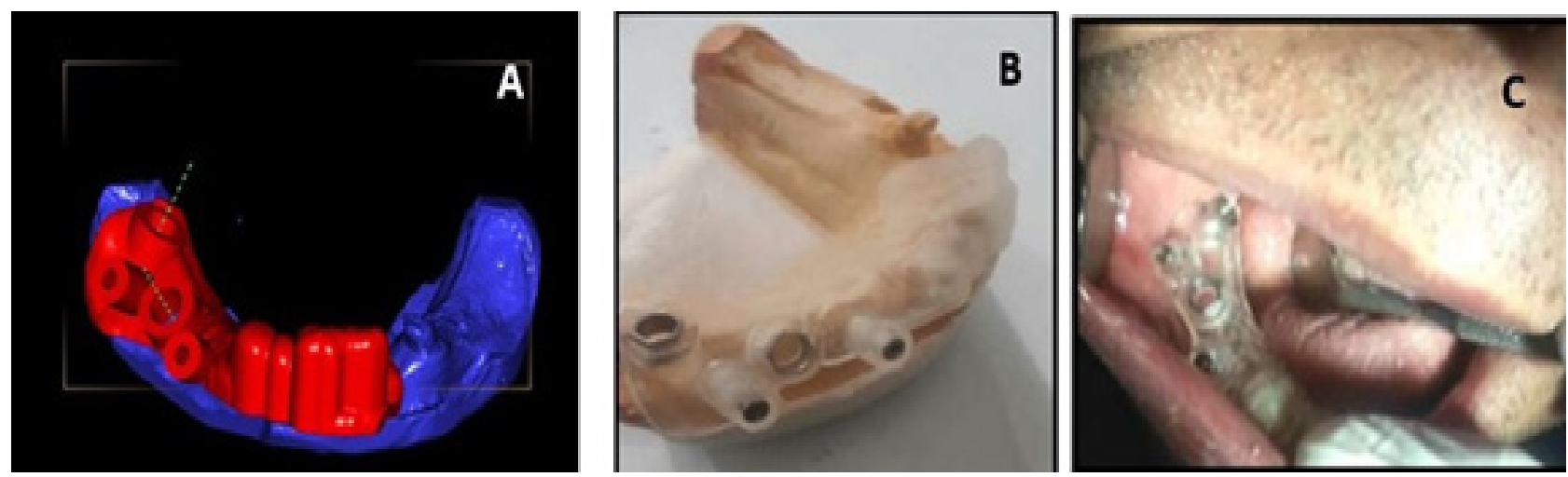

Figure 2: (A) Surgical guide virtual 3D design on DDS-Pro software. (B) the same surgical guide after being printed and seated on the cast with the sleeves incorporated. (C) the surgical guide after being checked in the patient's mouth.

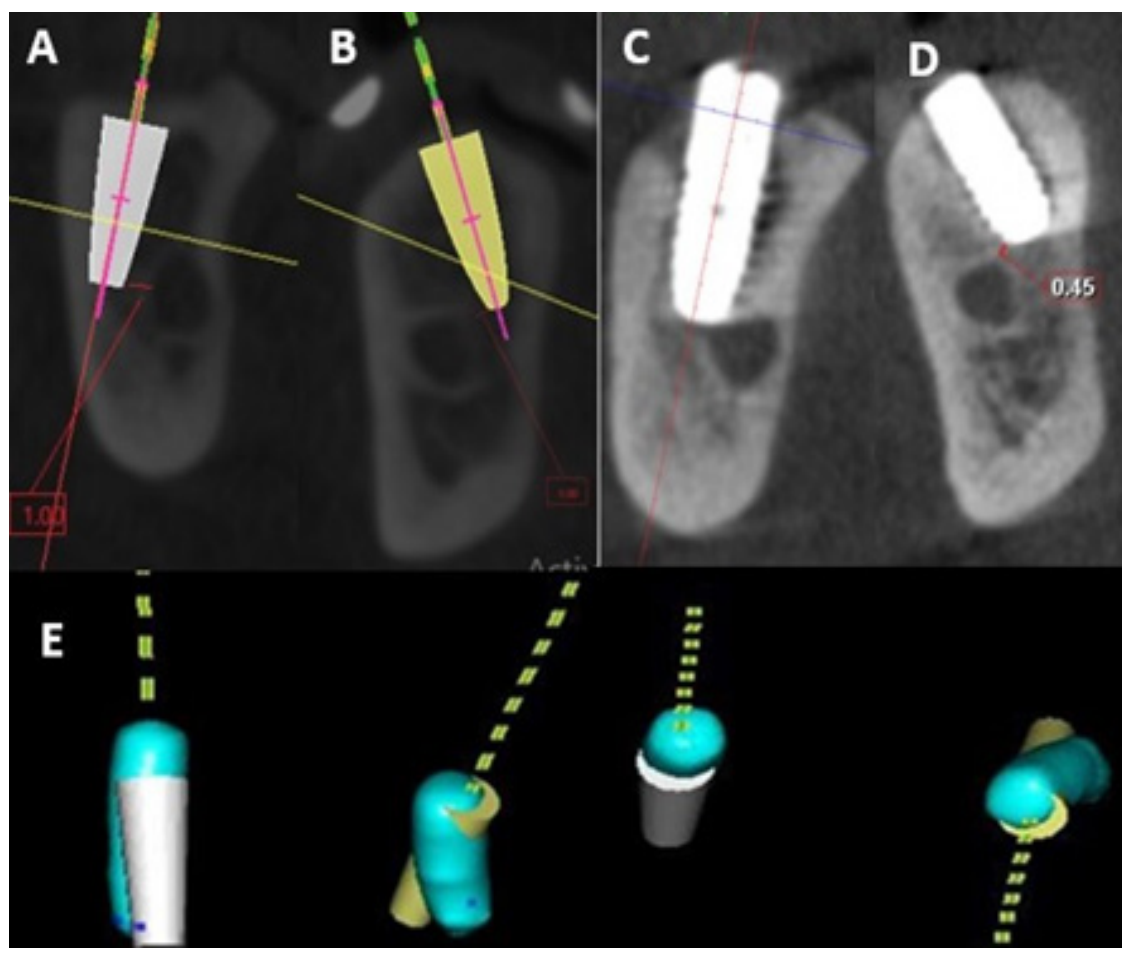

Figure 3: (A) A and B. The closet distance between the virtual implants and IAN C and D. The closet distance of the actual implants and the IAN after being placed by CASG, E. Registration of pre- and post-operative $\mathrm{CBCT}$ where the relation between the 3D segmented virtual and actual implants is clearly demonstrated (the teeth, bone tissues and IAN were hidden in these images just to highlight the variation in position between the virtual and actual implants from all the views) 


\section{A) Angular deviation (in degrees):}

To quantify the angulation between the long axes of virtual and actual implants.

\section{B) Cervical or Crestal deviation (in $\mathrm{mm}$ ):}

Measured as the horizontal distance between virtual and actual implants at the center of their platform.

\section{C) Apical deviation (in $\mathrm{mm}$ ):}

Measured as the horizontal distance between virtual and actual implants at the level of their apices.

\section{RESULTS}

Quantitative data of all the deviation values used in this study to assess the accuracy of CASG were presented as mean, standard deviation (SD) median and range values (Table 1). Wilcoxon signed-rank test was used for comparison between the closest distance of the implant to IAN in both virtual and actual implants in all the studied cases (Table 2). Statistical analysis was performed with IBM SPSS Statistics for Windows, Version 23.0. Armonk, NY: IBM Corp.

Table 1: Wilcoxon signed-rank test results for comparison between the closest distance of the implant to IAN in both virtual and actual implants in all the studied cases:

\begin{tabular}{cccccc}
\hline $\begin{array}{c}\text { Minimal } \\
\text { distance }(\mathrm{mm})\end{array}$ & Mean & SD & Median & Range & $P$ value \\
\hline Virtual implants & 1.7 & 0.56 & 1.75 & $1-2.5$ & $0.027^{*}$ \\
Actual implants & 0.72 & 0.25 & 0.7 & $0-1$ & \\
\hline
\end{tabular}

* Statistically significant at $p \leq 0.05$.

Table 2: Descriptive statistics for accuracy of computer assisted surgical guide using $\mathrm{CBCT}$ for implant placement in atrophied mandible $(\mathrm{n}=15)$ :

\begin{tabular}{lccccc}
\hline \multicolumn{1}{c}{ Deviation } & Mean & SD & Median & Range & $95 \%$ CI \\
\hline Angular $\left({ }^{\circ}\right)$ & 4.13 & 1.86 & 3.5 & $2.31-8$ & $3.1-5.16$ \\
Cervical $(\mathrm{mm})$ & 1.15 & 0.49 & 1.05 & $0.47-2$ & $0.88-1.43$ \\
Apical $(\mathrm{mm})$ & 1.32 & 0.63 & 1.2 & $0.58-2.5$ & $0.97-1.67$ \\
\hline
\end{tabular}

\section{DISCUSSION}

Restoring edentulous atrophied mandible with dental implants requires highly precise planning and conduction of the surgical procedures. recent advances in digital dentistry have claimed to offer such a high precession that promises the dentists with successful implant placement for effective restoration of edentulous areas without violation of the adjacent IAN or mental foramen. this was not possible previously without going through massive and coasty surgical procedures as grafting procedures, IAN transposition or IAN lateralization with the latter two surgeries commonly ending with neurosensory affection of IAN $^{[4,7}$ and 15$]$.
Computer guided surgical approach has attracted the attention of lots of researchers exploring its accuracy, applicability and predictability. Computer assisted surgical guide (CASG) is a stent fabricated from the 3D virtual plane made on the pre-operative $3 \mathrm{D}$ images of the patients for precise transfer of this virtual plane to the surgical theater. Stereolithography is a commonly used technology for production of such types of stent via rapid prototyping $^{[16,17]}$.

For that this study was designed to investigate the accuracy of fully guided implant placement using stereolithographic tooth and mucosa supported surgical guide in very critical situation of atrophied mandible with questionable amount of remaining alveolar bone obviating the need for bone grafting or nerve transposition surgery.

Assessment of the accuracy of CASG in this study was done by two means, first of them was the way commonly used by the researchers in such issue which is superimposition of pre and post-operative 3D images then calculation of the deviation between the virtual and actual implants, where the deviation measurements adopted in this study were selected based on their report by Marlière et al $2018^{[10]}$ as being the most commonly used measurements parameters in validation of CASG accuracy.

The deviation measurements in this method could be achieved either automatically with generation of color coded map for the deviation between the objects under investigations as made by different types of software reported in previous studies (Geomagic Control X v2018.0.1, 3D Systems ${ }^{[18]}$, Geomagic Qualify 2013 software $^{[19]}$, Geomagic WRAP 2016, Research Triangle Park, NC, USA ${ }^{[20]}$, Mimics, Materialise, Leuven, Belgium ${ }^{[13}$, 14 and 21$]$ ), or via manual calculation of the deviation at certain determined points as made in the current study using 'DDS-Pro software and other previous studies using On Demand3D, Cybermed Inc., Seoul, Korea ${ }^{[22]}$, Implant studio software19, Rhino 4.0, McNeel, Seattle, $\mathrm{WA}^{[23]}$ and Nobel Guide Validation 2.0.0.4 $4^{[24]}$.

However, a second way for validation of the accuracy of CASG was implemented in this study depending on the clinical significance anticipated from using the CASG in our cases, as preserving the IAN while properly restoring the edentulous atrophied mandible was the main concern in our cases, for that comparison between the virtual and actual implants' distance to the IAN was used in this study as a judge for success of reaching our goal from using CASG. This method has not been found in literature except in one study by Abd Alkader et al, 2020 $0^{[17\}}$.

Comparison between virtual and actual implants' distance to IAN showed a statistically significant difference, with a mean distance of $0.72 \pm 0.25 \mathrm{~mm}$ for actual implants and $1.7 \pm 0.56 \mathrm{~mm}$ for virtual implants, interpretation of this results raises an alarm that the distance of the actual implant to IAN is commonly lesser than that on virtual implants which could endanger the IAN if this was not 
considered in virtual planning. This finding actually coincides with that of Abd Alkader et al. 2020 $0^{[17]}$ who also found a significant difference in their comparison between virtual and actual implant distance to IAN as a means of CASG accuracy evaluation, but the reported values of this distance in their study $(4.30 \pm 2.69 \mathrm{~mm}$ for virtual implants and $3.88 \pm 2.23$ for actual implants) were higher than those in ours. The explanation for this difference is based on the fact that our study dealt with patients with atrophied mandible with questionable amount of remaining alveolar bone left for implant placement.

Before Comparing the part of our results concerned with deviation measurements between actual and planned implants with those reported in previous studies, care was taken not to include studies made in vitro or on cadaver heads as a systematic review with meta-analysis made to compare between accuracy of CASG in implant placement in different types of studies (clinical, in-vitro and cadaver studies) ${ }^{[25]}$ has found that clinical study recorded a significantly lower level of accuracy than that reported for in-vitro and cadaver studies. Only results of surgeries done on mandible were included in our comparison as the literature is full of debate that has not been yet solved regarding the effect of jaw type on the accuracy of the guided implant placement, some studies reported that accuracy in maxilla is higher than in mandible based on the higher surface area available for support of CASG in maxilla and the lower bone density in $\mathrm{it}^{[20,24}$ and 26$]$. On the other hands some other studies reported a lower level of accuracy in maxilla due to the higher amount of medullary bone which decreases the torque resistance compared to cortical bone, hence causes more obvious deviation in the position of the implant ${ }^{[23,27}$ and 28$]$.

Regarding the angular deviation reported in our study $\left(4.13 \pm 1.86^{\circ}\right)$ it was slightly higher than that reported for clinical studies by Bover-Ramos et al 2018 ${ }^{[25]}$ $\left(3.98 \pm 0.33^{\circ}\right)$ in their meta-analysis. However, it was very close to that of Nickenig et al. $2010^{[29]}\left(4.2 \pm 3.04^{\circ}\right)$ who conducted their fully guided surgeries on mandible, using CBCT as the imaging modality of choice, though they didn't report the type of support in the CASGs used. Also, close value of angular deviation was reported by Cassetta et al $2013^{[30]} 4.09 \pm 2.40^{\circ}$ for mucosa supported stereolithographic stents in mandible with fixation pins while a slightly higher value $\left(5.62 \pm 2.80^{\circ}\right)$ was recorded in the same study for the same type of CASG but without fixation pins, however it deserves mentioning that they utilized MSCT in their planning procedures. Also, Di Giacomo et al. 2012 ${ }^{[23]}$ reported slightly higher angular deviation $\left(5.37 \pm 3.98^{\circ}\right)$ in mucosa supported stent in mandible with fixation pins but the stent in this study was made by selective laser sintering technique based on CBCT images.

On the other hand, lower values of angular deviation were reported in mandibular guided implants as
Pettersson et al 2012[24] reported $2.16^{\circ}$ and Vieira et al 2013 ${ }^{[27]}$ reported $1.85 \pm 0.75^{\circ}$ angular deviation for mucosa supported stereolithographic surgical guides fixed with pins based on CBCT images.

When it comes to the horizontal deviation between actual and planned implant cervically; this study recorded a mean deviation of $1.15 \pm 0.49 \mathrm{~mm}$ which was very close to the mean cervical deviation for clinical studies included in Bover-Ramos' meta-analysis $(1.1 \pm 0.09)$, it was also close to the those recorded by Di Giacomo et al. 2012 $2^{[23]}$ $(1.26 \pm 0.66 \mathrm{~mm})$, Vieira et al $2013^{[27]}(1.42 \pm 0.71 \mathrm{~mm})$ and Abd Alkader et al. $2020^{[17]}(0.96 \pm 0.7 \mathrm{~mm})$ in mandibular implants placed by mucosa supported stereolithographic CASG fixed with pins based on CBCT images. However, this cervical deviation is considered slightly higher than those recorded by Pettersson et al 2012 $2^{[24]}(0.8 \mathrm{~mm})$ in mandibular implants placed by mucosa supported selective laser sintering CASG fixed with pins based on CBCT images, and slightly lower than that recorded by Cassetta et al $2013^{[30]}(1.64 \pm 71 \mathrm{~mm})$ in mandibular implants placed by mucosa supported stereolithographic CASG fixed with pins based on MSCT images.

Concerning the apical horizontal deviation, a mean of $1.32 \pm 0.63 \mathrm{~mm}$ was recorded in the current study which -like cervical deviation- was very close to that reported in Bover-Ramos et al 2018 $8^{[25]}$ meta-analysis $(1.4 \pm 0.12 \mathrm{~mm})$ and to what was recorded by Vieira et al $2013^{[27]}(1.57 \pm 0.84 \mathrm{~mm})$, but unlike cervical deviation it was lower than that recorded by Di Giacomo et al. $2012^{[23]}(1.75 \pm 0.99 \mathrm{~mm})$ and Cassetta et al 2013 $3^{[30]}$ $(2.25 \pm 0.88 \mathrm{~mm})$.

From the previous comparisons we can find that although high coincidence was found between our results and those of meta-analysis collecting lot of clinical studies addressing the same issue, still some diversity exists between our results and those of some other studies but within a very limited level of difference. This diversity could be attributed to a lot of factors that have an effect on the reported CASG accuracy. Although a very limited number of researches were found addressing these factors ${ }^{[10,30]}$ but we can summarize some of them based on our literature reviewing.

The start comes from patient selection- wither fully or partially edentulous patient- which in turn affects the type of support to be used for surgical guide, a big unsolved debate was found in literature regarding which type of support provides the highest accuracy of static computer guided implant procedures ${ }^{[31-33]}$. Moreover, patient's selection is also affecting the image acquisition technique to be used wither single or dual scan technique, with the later inviting more chances for discrepancies and inaccuracy due to addition of image fusion procedures ${ }^{[20,34]}$.

The image acquisition, manipulation and processing procedures could introduce a source of inaccuracy that 
might be up to $0.5 \mathrm{~mm}^{[20,35]}$. Besides, the software used for virtual implant planning and surgical guide design could be another source of discrepancies that varies from $0.1-0.2 \mathrm{~mm}$ if not properly configured ${ }^{[36]}$. In addition, the level of guidance obtained wither full or half guidance has been found to be significantly affecting the accuracy of the guided implant procedure with fully guided stents providing higher accuracy than half guided ones or those guiding only pilot drill ${ }^{[10,20,22,25,29]}$. The precession of prototyping machine used wither by stereolithography or selective laser sintering and the properties of the materials used in surgical guide generation were also found to be affecting the final accuracy of the $\mathrm{CASG}^{[37,38]}$. Last but not least the level of experience of the operator has an effect on the final reported accuracy of computer guided surgical procedures $^{[39]}$. Now it is clear that the final accuracy of CASG represents an interplay between several factors that should all be properly considered for obtaining the best possible level of accuracy in each individual case.

\section{CONCLUSION}

Although a significant difference was found between virtual and actual implants distance to IAN, the linear and angular deviation between them were well accepted and within the range reported by most of similar clinical studies with less crucial situations, which enhance using fully guided stereolithographic CASG for implant placement with tilted position in posterior atrophied mandible but with considering at least $2 \mathrm{~mm}$ distance between virtual implant and IAN in planning procedures.

\section{CONFLICT OF INTEREST}

The authors declare no conflict of interest.

\section{REFERENCES}

1. Misch CE. Contemporary Implant Dentistry. $2^{\text {nd }}$ ed. St. Louis, Missouri. Mosby; 1993.

2. Mittal Y, Jindal G, Garg S. Bone manipulation procedures in dental implants. Indian $\mathrm{J}$ Dent. 2016; 7: 86 - 94.

3. Cullum D. Advances in bone manipulation: Part 2. Osteomobilization for horizontal and vertical implant site development. SORMS. 2010; 18: $1-44$.

4. Basa S, Varol A, Turker N. Alternative bone expansion technique for immediate placement of implants in the edentulous posterior mandibular ridge: A clinical report. Int $\mathrm{J}$ Oral Maxillofac Implants. 2004; 19: $554-8$.

5. Abayev B, Juodzbalys G. Inferior alveolar nerve lateralization and transposition for dental implant placement. Part I: a systematic review of surgical techniques. J Oral Maxillofac Res. 2015; 6: e2.

6. Suzuki D, Bassi AP, Lee HJ, Alcântara PR, de Sartori IM, Luvizuto ER, Faco EF, Faot F. Inferior alveolar nerve lateralization and implant placement in atrophic posterior mandible. J Craniofac Surg. 2012; 23: e347 - 9 .

7. Kan JY, Lozada JL, Goodacre CJ, Davis WH, Hanisch O. Endosseous implant placement in conjunction with inferior alveolar nerve transposition: an evaluation of neurosensory disturbance. Int J Oral Maxillofac Implants. 1997; 12: 463 - 71.

8. Guerrero ME, Jacobs R, Loubele M, et al. State-ofthe-art on cone beam CT imaging for preoperative planning of implant placement. Clin Oral Investig 2006; 10: $1-7$.

9. Mora MA, Chenin DL, Arce RM. Software tools and surgical guides in dental-implant-guided surgery. Dent Clin North Am. 2014; 58: 597.

10. Marlière DAA, Demètrio MS, Picinini LS, Oliveira RG, Netto HDMC. Accuracy of computer-guided surgery for dental implant placement in fully edentulous patients: A systematic review. Eur J Dent. 2018; 12: 153 - 160.

11. Sghaireen MG, Srivastava KC, Shrivastava D, Ganji KK, Patil SR, Abuonq A, Mousa MA, DarOdeh N, Sghaireen GM, Kamal MA, Alam MK. A CBCT Based Three-Dimensional Assessment of Mandibular Posterior Region for Evaluating the Possibility of Bypassing the Inferior Alveolar Nerve While Placing Dental Implants. Diagnostics (Basel). 2020 14; 10: 406.

12. Wang TY, Kuo PJ, Fu E, Kuo HY, Nie-Shiuh Chang N, Fu MW, Shen EC, Chiu HC. Risks of angled implant placement on posterior mandible buccal/lingual plated perforation: A virtual immediate implant placement study using CBCT. J Dent Sci. 2019 14: 234 - 240.

13. Stübinger S, Buitrago Tellez C, Cantelmi G. Deviations between placed and planned implant positions: An accuracy pilot study of skeletally supported stereolithographic surgical templates. Clin Implant Dent Relat Res 2014; 16: 54051.

14. Ochi M, Kanazawa M, Sato D, Kasugai S, Hirano $\mathrm{S}$, Minakuchi S, et al. Factors affecting accuracy of implant placement with mucosa supported stereolithographic surgical guides in edentulous mandibles. Comput Biol Med 2013; 43: 165360. 
15. Shotwell JL, Billy EJ, Wang HL, Oh TJ. Implant surgical guide fabrication for partially edentulous patients. J Prosthet Dent 2005; 93: 294 - 7.

16. Lal K, White GS, Morea DN, Wright RF. Use of stereolithographic templates for surgical and prosthodontic implant planning and placement. Part II. A clinical report. J Prosthodont 2006; 15: 117 - 22.

17. Abd Alkader WM, Fahmy MH, ElAshwah AA. Accuracy of guided implant insertion in mandibular free-end saddle areas using stereolithographic surgical stent. Alexandria Dental Journal. 2020 1; 45: 117 - 22.

18. Son K, Huang MY, Lee KB. A method to evaluate the accuracy of dental implant placement without postoperative radiography after computer-guided implant surgery: A dental technique. J Prosthet Dent. 2020; 123: 661 - 666.

19. Cristache CM, Gurbanescu S. Accuracy evaluation of a stereolithographic surgical template for dental implant insertion using 3D superimposition protocol. International journal of dentistry. 2017; 7: 2017.

20. De Santis D, Malchiodi L, Cucchi A, Cybulski A, Verlato G, Gelpi F, Nocini PF. The Accuracy of Computer-Assisted Implant Surgery Performed Using Fully Guided Templates versus PilotDrill Guided Templates. BioMed Research International. 2019 8; 2019.

21. D'haese J, Van De Velde T, Elaut L, De Bruyn H. A prospective study on the accuracy of mucosally supported stereolithographic surgical guides in fully edentulous maxillae. Clinical implant dentistry and related research. 2012; 14: 293 - 303.

22. Ahmed MF, AbdelHamid AM, AlAbbasy FH. Accuracy of implant placement using two different types of $\mathrm{CAD} / \mathrm{CAM}$ surgical guides (an invitro study). Alexandria Dental Journal. 2019 1; 44: 28 - 33.

23. Di Giacomo GA, da Silva JV, da Silva AM, Paschoal GH, Cury PR, Szarf G. Accuracy and complications of computer $\square$ designed selective laser sintering surgical guides for flapless dental implant placement and immediate definitive prosthesis installation. Journal of periodontology. 2012; 83: 410 - 9 .

24. Pettersson A, Komiyama A, Hultin M, Näsström $\mathrm{K}$, Klinge B. Accuracy of virtually planned and template guided implant surgery on edentate patients. Clinical implant dentistry and related research. 2012; 14: 527 - 37.

25. Bover-Ramos F, Viña-Almunia J, CerveraBallester J, Peñarrocha-Diago M, García-Mira B. Accuracy of Implant Placement with ComputerGuided Surgery: A Systematic Review and MetaAnalysis Comparing Cadaver, Clinical, and In Vitro Studies. International Journal of Oral and Maxillofacial Implants. 2018; 1 : 33 (1).

26. Cassetta M, Stefanelli LV, Giansanti M, Di Mambro A, Calasso S. Depth deviation and occurrence of early surgical complications or unexpected events using a single stereolithographic surgi-guide. Int J Oral Maxillofac Surg. 2011; 40: 1377 - 87.

27. Vieira DM, Sotto Maior BS, Barros CA, Reis ES, Francischone CE. Clinical accuracy of flapless computer guided surgery for implant placement in edentulous arches. Int J Oral Maxillofac Implants 2013; 28: 134751 .

28. Cassetta M, Giansanti M, Di Mambro A, Stefanelli LV. Accuracy of positioning of implants inserted using a mucosa supported stereolithographic surgical guide in the edentulous maxilla and mandible. Int $\mathbf{J}$ Oral Maxillofac Implants 2014; 29: 10718.

29. Nickenig HJ, Wichmann M, Hamel J, Schlegel KA, Eitner S. Evaluation of the difference in accuracy between implant placement by virtual planning data and surgical guide templates versus the conventional free-hand method a combined in vivo - in vitro technique using cone-beam CT (Part II). J Craniomaxillofac Surg. 2010; 38: 488 - 93.

30. Cassetta M, Di Mambro A, Giansanti M, Stefanelli LV, Cavallini C. The intrinsic error of a stereolithographic surgical template in implant guided surgery. Int J Oral Maxillofac Surg. 2013; 42: $264-75$.

31. Cassetta M, Stefanelli LV, Giansanti M, Di Mambro A, Calasso S. Accuracy of a computeraided implant surgical technique. Int J Periodontics Restorative Dent. 2013; 33: 317 - 25.

32. Ozan O, Turkyilmaz I, Ersoy AE, McGlumphy EA, Rosenstiel SF. Clinical accuracy of 3 different types of computed tomographyderived stereolithographic surgical guides in implant placement. J Oral Maxillofac Surg. 2009; 67: 394 - 401. 
33. Ersoy AE, Turkyilmaz I, Ozan O, McGlumphy EA. Reliability of implant placement with stereolithographic surgical guides generated from computed tomography: clinical data from 94 implants. J Periodontol. 2008; 79: 1339 - 45.

34. Verhamme LM, Meijer GJ, Boumans T, de Haan AF, Bergé SJ, Maal TJ. A clinically relevant accuracy study of computer $\square$ planned implant placement in the edentulous maxilla using mucosa $\square$ supported surgical templates. Clinical implant dentistry and related research. 2015; 17: 343 - 52.

35. Reddy MS, Mayfield Donahoo T, Vanderven FJ, Jeffcoat MK. A comparison of the diagnostic advantages of panoramic radiography and computed tomography scanning for placement of root form dental implants. Clin Oral Implants Res 1994; 5: 22938.

36. Van Steenberghe D, Naert I, Andersson M, Brajnovic I, Van Cleynenbreugel J,
Suetens $\mathrm{P}$, et al. A custom template and definitive prosthesis allowing immediate implant loading in the maxilla: A clinical report. Int J Oral Maxillofac Implants 2002; 17: 66370.

37. Stumpel L J. Deformation of stereolithographically produced surgical guides: an observational case series report. Clin Implant Dent Relat Res. 2012; 14: 442 - 53 .

38. Ibrahim D, Broilo TL, Heitz C, et al. Dimensional error of selective laser sintering, three-dimensional printing and PolyJet models in the reproduction of mandibular anatomy. J Craniomaxillofac Surg 2009; 37: 167 - 173 .

39. Rungcharassaeng, K., Caruso, J. M., Kan, J. Y. K., Schutyser, F., and Boumans, T. Accuracy of computer-guided surgery: A comparison of operator experience. The Journal of Prosthetic Dentistry. 2015; 114: 407 - 413. 